\title{
Substrate Availability Limits Human Skeletal Muscle Oxidative ATP Regeneration at the Onset of Ischemic Exercise
}

\author{
James A. Timmons, ${ }^{\star}$ Thomas Gustafsson, ${ }^{\ddagger}$ Carl Johan Sundberg, ${ }^{\ddagger}$ Eva Jansson, ${ }^{\S}$ Eric Hultman, ${ }^{*}$ Lenart Kaijser, ${ }^{\S}$ \\ Jolanta Chwalbinska-Moneta, $\$$ Dimutri Constantin-Teodosiu, ${ }^{*}$ lan A. Macdonald, ${ }^{\star}$ and Paul L. Greenhaff* \\ *Department of Physiology and Pharmacology, University Medical School, Queen's Medical Centre, Nottingham, United Kingdom; and \\ ${ }^{\ddagger}$ Department of Physiology and Pharmacology, and ${ }^{\S}$ Department of Clinical Physiology, Huddinge Hospital, Karolinska Institute, \\ Stockholm, Sweden
}

\begin{abstract}
We have demonstrated previously that dichloroacetate can attenuate skeletal muscle fatigue by up to $35 \%$ in a canine model of peripheral ischemia (Timmons, J.A., S.M. Poucher, D. Constantin-Teodosiu, V. Worrall, I.A. Macdonald, and P.L. Greenhaff. 1996. J. Clin. Invest. 97:879-883). This was thought to be a consequence of dichloroacetate increasing acetyl group availability early during contraction. In this study we characterized the metabolic effects of dichloroacetate in a human model of peripheral muscle ischemia. On two separate occasions (control-saline or dichloroacetate infusion), nine subjects performed 8 min of single-leg knee extension exercise at an intensity aimed at achieving volitional exhaustion in $\sim 8 \mathrm{~min}$. During exercise each subject's lower limbs were exposed to $50 \mathrm{mmHg}$ of positive pressure, which reduces blood flow by $\sim 20 \%$. Dichloroacetate increased resting muscle pyruvate dehydrogenase complex activation status by threefold and elevated acetylcarnitine concentration by fivefold. After $3 \mathrm{~min}$ of exercise, phosphocreatine degradation and lactate accumulation were both reduced by $\sim 50 \%$ after dichloroacetate pretreatment, when compared with control conditions. However, after $8 \mathrm{~min}$ of exercise no differences existed between treatments. Therefore, it would appear that dichloroacetate can delay the accumulation of metabolites which lead to the development of skeletal muscle fatigue during ischemia but does not alter the metabolic profile when a maximal effort is approached. (J. Clin. Invest. 1998. 101:79-85.) Key words: acetylcarnitine • pyruvate dehydrogenase complex • ischemia • phosphocreatine • lactate
\end{abstract}

\section{Introduction}

The metabolic effects of dichloroacetate (DCA) $)^{1}$ administration have been examined in humans at rest $(1,2)$ and during

Address correspondence to Dr. James A. Timmons, Discovery Biology III, Central Research, Pfizer Limited, Sandwich, Kent CT13 9NJ, United Kingdom. FAX: 44-1304-618444; E-mail: James_Timmons@ sandwich.pfizer.com

Received for publication 9 July 1997 and accepted in revised form 17 October 1997.

1. Abbreviations used in this paper: DCA, dichloroacetate; dm, dry muscle; PCr, phosphocreatine; PDC, PDCa, pyruvate dehydrogenase complex and active PDC.

J. Clin. Invest.

(C) The American Society for Clinical Investigation, Inc. 0021-9738/98/01/0079/07 \$2.00

Volume 101, Number 1, January 1998, 79-85

http://www.jci.org submaximal and maximal exercise $(3,4)$. These studies have consistently demonstrated that DCA can reduce blood lactate concentration at rest and during exercise. Furthermore, it has been reported that DCA administration can increase maximal oxygen uptake $\left(\dot{\mathrm{V}}_{2 \max }\right)$ and maximal exercise performance under double-blind, randomized, cross-over experimental conditions (4). To date, no study has directly examined the effects of DCA on human skeletal muscle metabolism, such that the mechanisms for the observed improvements in exercise capacity remain unexplained. However, using a canine model it has been demonstrated that DCA reduced the extent of skeletal muscle fatigue by $\sim 35 \%$ during 20 min of ischemic contraction (5) and that this improvement was associated with a decreased reliance on ATP regeneration derived from substrate level phosphorylation (phosphocreatine [PCr] hydrolysis and glycolysis). It was hypothesized (5) that these alterations in skeletal muscle metabolism may have significant implications for improving exercise tolerance in peripheral vascular disease $(6,7)$ and heart failure patients (8). It is important to note that DCA administration does not alter muscle vascular resistance or blood flow to skeletal muscle during contraction $(5,9)$.

It has been demonstrated, more recently, that the improvement in contractile function was preceded by a reduction in the rate of $\mathrm{PCr}$ degradation during the early stages of contraction in a canine model of ischemia (9), thus reflecting a faster onset of oxidative phosphorylation during this period (10). The consequences of this were a reduction in muscle glycogenolysis, lactate accumulation, and skeletal muscle fatigue and the provision of a rationale for the observed improvement in exercise capacity in humans.

Pyruvate dehydrogenase complex (PDC) catalyzes the decarboxylation of pyruvate, forming acetyl-CoA, which can subsequently be used by the tricarboxylic acid (TCA) cycle or the carnitine acetyltransferase reaction. DCA activates PDC in resting skeletal muscle by inhibiting the kinase responsible for phosphorylating and thus inactivating the enzyme complex (11). This maximizes the flux of pyruvate through the PDC reaction immediately at the onset of contraction. Infusion of DCA also results in the acetylation of $\sim 90 \%$ of the intramuscular carnitine pool at rest (9), providing a readily available pool of substrate for mitochondrial oxidation, since the maximal catalytic activity of the acetylcarnitine transferase/translocase system greatly exceeds that of PDC (12). Although acetylCoA production appears to exceed its rate of utilization, when assessed during steady state contractions (13-15), our previous work has demonstrated that acetylcarnitine concentration falls during the onset of contraction, after DCA administration, suggesting that flux through PDC fails to meet the demand of the TCA cycle for oxidative substrate during this period (5). Thus it seems likely that under normal conditions acetyl-CoA production during this period follows a biphasic response, whereby a period of limited supply is followed by an excess 
rate of production and hence the observed accumulation of acetylcarnitine once steady state is achieved.

In this study, we have examined the effects of DCA on the metabolic response of human skeletal muscle during single-leg knee extension ischemic exercise at an intensity that would result in volitional fatigue in $\sim 8 \mathrm{~min}$. We were interested to establish whether the metabolic responses observed previously in the canine model $(5,9)$ could be reproduced during voluntary exercise, thereby demonstrating that intracellular substrate availability limits the onset of oxidative ATP regeneration in human skeletal muscle. The physiological and clinical implications of this are clearly evident during skeletal muscle ischemia $(5,9)$, such as that experienced in peripheral vascular disease and heart failure (6-8).

\section{Methods}

Subjects. Nine healthy male volunteers participated in this study which was approved by the Ethics Committee of the Karolinska Institute. The average (range) age, height, body mass, and background physical activity levels of the subjects were 22 (21-25) yr, 182 (176186) $\mathrm{cm}, 77(70-89) \mathrm{kg}$, and $4.5(2-8) \mathrm{h} \cdot \mathrm{wk}^{-1}$ of moderate exercise, respectively. Before the study, the experimental protocol was explained to all subjects and their written consent was obtained. DCA was purchased from Sigma Chemical Co. (Stockholm, Sweden) and was prepared for sterile infusion by Apoteksbolaget (Umeå, Sweden). The aqueous solution was sterilized by filtration, had a $\mathrm{pH}$ of 7.0, and osmolality of $550 \mathrm{mosmol} / \mathrm{kg}$.

Exercise model. The study was conducted at the Department of Environmental Physiology at the Karolinska Institute and used the hyperbaric pressure chamber technique of Eiken and Bjurstedt (16), which involves a supraatmospheric pressure being applied to the lower body to reduce muscle blood flow during exercise (for a diagram see reference 17). Each subject was positioned supine with his legs inserted through a rubber diaphragm into the chamber, with the rubber diaphragm sealed around the proximal part of the subject's lower limbs. The subject's shoulders were restrained using padded struts to prevent displacement of his body out of the chamber when the supraatmospheric pressure was applied. A 50-mmHg pressure gradient was used which results in an $\sim 20 \%$ reduction in muscle blood flow during cycle exercise (18).

At least $1 \mathrm{wk}$ before the first experiment, each subject was thoroughly familiarized with the exercise model and procedures which consisted of single-leg knee extension using a modified electrically braked cycle ergometer at an individually fixed work load. During familiarization, the maximal one-legged performance capacity was determined during ischemic exercise conditions. The work load started with $4 \mathrm{~min}$ at $5 \mathrm{~W}$ and was increased by $5 \mathrm{~W}$ at 1 -min intervals to fatigue. During the experiment the mean work load was $20 \pm 2 \mathrm{~W}$ (60 $\mathrm{rev} \cdot \mathrm{min}^{-1}$ ) with this corresponding to $\sim 65 \%$ of the final work load achieved during the 1-min incremental exercise protocol. Overall, this represented a work load that could be maintained, without a reduction in cadence occurring $\left(<60 \mathrm{rev} \cdot \mathrm{min}^{-1}\right)$, for a maximum of $\sim 8$ min when the pressure inside the chamber was elevated (blood flow reduced).

Study protocol. Each subject rested in a supine position and a 21gauge cannula was placed in an antecubital vein of each arm. They then received, in a randomized, double-blind, cross-over fashion, 50 $\mathrm{mg} \cdot \mathrm{kg}^{-1}$ body mass DCA $\left(50 \mathrm{mg} \cdot \mathrm{ml}^{-1}\right.$, sodium salt, $\left.\mathrm{pH} 7.0\right)$ or an equivalent volume of saline, via intravenous infusion over a 30 -min period, commencing $1 \mathrm{~h}$ before the start of exercise. After the $30 \mathrm{~min}$ of infusion, the subject took up the position detailed above, with his legs inside the hyperbaric chamber. 5 min before the onset of exercise, a resting muscle biopsy from vastus lateralis was taken using a Bergström needle (19) and blood samples were obtained. Blood pressure and heart rate were determined manually every 15 min through- out the infusion period, while the subject was resting supine. During exercise, heart rate was derived continuously from an ECG monitor and blood pressure was obtained every 2 min using automated sphygmomanometry (exercise monitor 1165; Criticon, Stockholm, Sweden).

A leg of each subject was then attached to the pedal crank of the modified cycle ergometer, via a lever system. The site of attachment was half way between the patella and the ankle. The pressure was then increased in the chamber by $50 \mathrm{mmHg}$ and the subjects were then asked to exercise at their individually determined work load for $8 \mathrm{~min}$. Each voluntary contraction extended the leg from $70^{\circ}$ to $150^{\circ}$ (vertical to horizontal at the knee). Flexion was performed passively using the ergometer flywheel momentum to reposition the leg for the next extension. The protocol was interrupted for $30 \mathrm{~s}$ after $3 \mathrm{~min}$ of exercise and a second muscle biopsy was obtained. The subject then resumed exercise for a further $5 \mathrm{~min}$ of exercise after which a third biopsy was obtained (total exercise time $=8 \mathrm{~min}$ ). $7-10 \mathrm{~d}$ later, the same protocol was repeated with the same leg being used, but on this occasion the alternative infusate was administered.

Blood and muscle sample collection and analysis. Forearm venous blood was obtained at predetermined intervals throughout the experiment for the assessment of blood glucose (reflectance monitor) and lactate concentrations. Blood lactate was assessed in perchloric acid extracts modified for use with a fluorescence spectrophotometer (see reference 33). Muscle biopsy samples were freeze-dried, dissected free from visible blood and connective tissue, powdered, and $\sim 10 \mathrm{mg}$ was extracted in $0.5 \mathrm{M}$ perchloric acid containing $1 \mathrm{mM}$ EDTA. After centrifugation, the supernatant was neutralized with $2.2 \mathrm{M} \mathrm{KHCO}_{3}$ and used for spectrophotometric determination of ATP, PCr, creatine, and lactate (20). The extract was also used for the radioisotopic determination of acetylcarnitine (21). Briefly, for the determination of acetylcarnitine, the acetyl group was transferred to $\mathrm{CoASH}$ in a reaction catalyzed by carnitine acetyltransferase to form acetyl-CoA. Acetyl$\mathrm{CoA}$ so formed was condensed with $\left[{ }^{14} \mathrm{C}\right]$ oxaloacetate to form $\left[{ }^{14} \mathrm{C}\right] \mathrm{ci}-$ trate which was then measured. Freeze-dried muscle powder was also used for the determination of muscle glycogen (20). Muscle powder was digested in $0.5 \mathrm{M} \mathrm{NaOH}$ at $80^{\circ} \mathrm{C}$ for 20 min and neutralized with HCl-citrate buffer ( $\mathrm{pH} 4.9$ ). This extract was then hydrolyzed using amyloglucosidase and, after centrifugation, the supernatant was analyzed for glucose. Intramuscular metabolites were corrected for total muscle creatine concentration. In the resting muscle biopsy procedures, sufficient muscle was obtained to assess PDC activation status (PDCa), and wet samples were stored under liquid nitrogen until analysis was performed. Briefly, activity was measured by the addition of NaF and DCA to the extraction buffer and then the rate of production of acetyl-CoA, when incubated with pyruvate, was measured as described previously (22).

Statistics and calculations. All data are reported as mean \pm SE. Muscle metabolite comparisons between treatments (saline-control and DCA infusion) were made using repeated-measures ANOVA on the changes from metabolite concentrations at rest (23). Blood metabolite comparisons between treatments at each time point were carried out also using repeated-measures ANOVA. When a significant $F$ ratio was found, a Student's paired $t$ test was used to locate the time point(s) at which any differences between treatments reached significance, which was accepted at the 5\% level. ATP production derived from substrate level phosphorylation was estimated from: $\Delta \mathrm{PCr}+$ $(1.5 \times \Delta$ lactate $)+(2 \times \Delta$ ATP $)$, where $\Delta$ is the difference between rest and contraction concentrations (24).

\section{Results}

Cardiovascular and blood metabolite parameters. DCA infusion did not significantly alter blood glucose concentration at any time during the experiment, nor did DCA infusion alter heart rate or mean arterial pressure during the infusion protocol (Table I). However, DCA infusion did reduce resting and 
Table I. Venous Blood Glucose Concentration and Cardiovascular Parameters Taken at Rest and after 8 min of One-legged Knee Extension Ischemic Exercise

\begin{tabular}{|c|c|c|c|c|c|c|c|c|}
\hline & \multicolumn{4}{|c|}{ Control } & \multicolumn{4}{|c|}{ Dichloroacetate } \\
\hline & Pre-infusion & Post-infusion & Pre-biopsy & End & Pre-infusion & Post-infusion & Pre-biopsy & End \\
\hline Glucose & $4.9 \pm 0.1$ & $4.8 \pm 0.1$ & $4.7 \pm 0.2$ & $4.9 \pm 0.2$ & $5.1 \pm 0.2$ & $5.0 \pm 0.2$ & $4.6 \pm 0.1$ & $4.8 \pm 0.3$ \\
\hline HR & $60 \pm 2$ & $59 \pm 3$ & $61 \pm 2$ & $172 \pm 12$ & $59 \pm 4$ & $62 \pm 4$ & $63 \pm 3$ & $169 \pm 12$ \\
\hline MAP & $85 \pm 2$ & $84 \pm 2$ & $87 \pm 2$ & NA & $82 \pm 2$ & $83 \pm 1$ & $84 \pm 1$ & NA \\
\hline
\end{tabular}

Venous samples taken immediately before infusion (saline control or dichloroacetate), 30 min after (postinfusion), just before the onset of the exercise period, and at the end of the 8-min exercise protocol. Data are mean \pm SE. Glucose units are mmol-liter ${ }^{-1}$, heart rate $(H R)$ is beats per min, and mean arterial pressure $(M A P)$ is $\mathrm{mmHg}$. $N A$, Not assessed.

exercise blood lactate concentration significantly at all time points when compared with the control condition (Fig. 1).

Resting muscle metabolism. There were no differences in muscle ATP, PCr, lactate, and glycogen concentrations between control and DCA conditions at rest (Table II). However, DCA did result in a marked increase in acetylcarnitine concentration before the onset of ischemic exercise $(P<0.01)$. In addition, a threefold increase in the activation status of skeletal muscle PDC was achieved after DCA (Fig. 2, $P<0.01$ ).

Changes after 3 min of ischemic exercise. There was a small reduction in the ATP concentration after 3 min of ischemic exercise in the control condition, whereas ATP concentration remained unchanged after DCA pretreatment $(P=0.09)$. PCr concentration was higher after DCA administration and this represented an $\sim 50 \%$ reduction in $\mathrm{PCr}$ degradation during the first 3 min of exercise (Fig. 3, $P<0.01$ ). Similarly, muscle

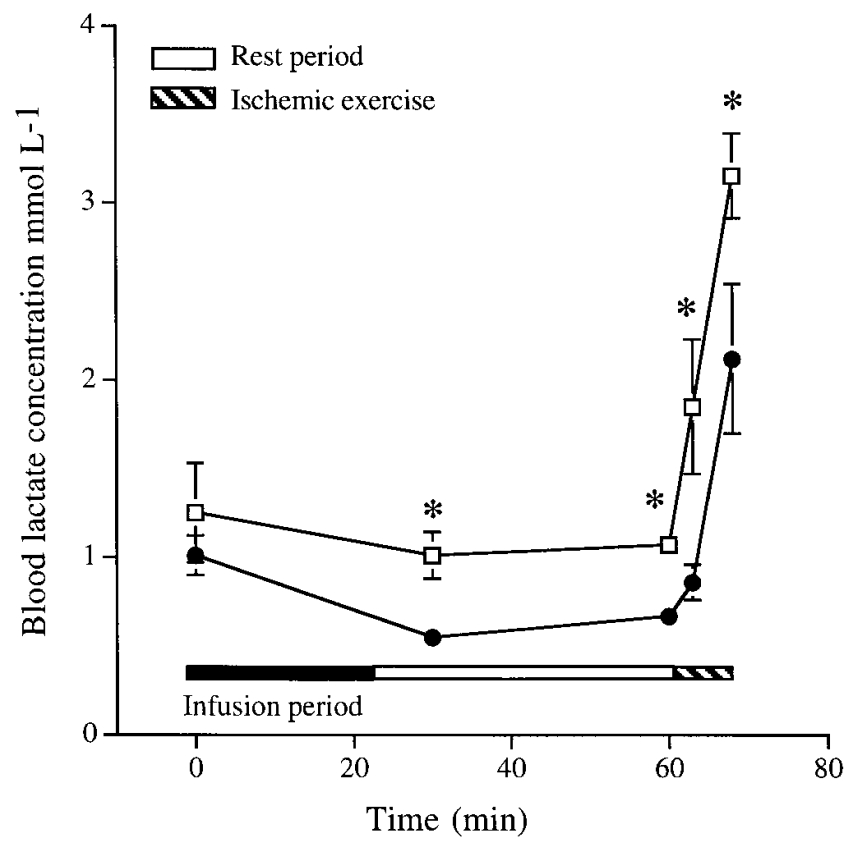

Figure 1. Venous blood lactate concentration before and after pretreatment with saline (open boxes) or DCA (filled circles, $50 \mathrm{mg} \cdot \mathrm{kg}^{-1}$ body mass infused over $30 \mathrm{~min}) . * P<0.05$, indicates DCA significantly lower than the corresponding control time point. Values are mean \pm SE. lactate accumulation was reduced by $\sim 50 \%$ after DCA administration (Fig. 4, $P<0.05$ ). There was also a $50 \%$ reduction in the amount of ATP regenerated from substrate level phosphorylation $(\mathrm{DCA}=57.1 \pm 14.0$ vs. control $=115.2 \pm 13.4$ mmol ATP $\cdot \mathrm{kg}^{-1}$ dry muscle $\left.[\mathrm{dm}], P<0.01\right)$. The changes in acetylcarnitine concentration differed significantly between the treatments (Fig. 5), with an increase in concentration during the control visit and a decrease during the DCA visit when compared with the respective resting concentrations $(P<$ $0.01)$. There was no significant difference in the rate of glycogenolysis between treatments.

Changes after 8 min of ischemic exercise. There were significant reductions in ATP concentration in both the control visit $(\sim 30 \%)$ and the DCA visit $(\sim 15 \%)$, but the extent of this fall did not differ significantly between treatments (control = $9.0 \pm 2.7$ and DCA $=4.4 \pm 2.5 \mathrm{mmol} \cdot \mathrm{kg}^{-1} \mathrm{dm}, P=0.1$ ). Both conditions demonstrated almost complete degradation of $\mathrm{PCr}$ stores with no difference being evident between groups (Table II and Fig. 3). In addition, muscle lactate accumulation (Fig. 4) and muscle glycogenolysis (control $=146.0 \pm 42.5$ and DCA $=$ $116.5 \pm 34.5 \mathrm{mmol} \cdot \mathrm{kg}^{-1} \mathrm{dm}$ ) did not significantly differ between treatments. ATP regeneration derived from substrate level phosphorylation was $178 \pm 24.8 \mathrm{mmol} \mathrm{ATP} \cdot \mathrm{kg}^{-1} \mathrm{dm}$ in the con-

Table II. Skeletal Muscle Metabolites in Saline and DCA Pretreated Subjects, at Rest and after 3 and 8 min of Ischemic Exercise

\begin{tabular}{|c|c|c|c|c|c|c|}
\hline & \multicolumn{2}{|c|}{ Resting } & \multicolumn{2}{|c|}{$3 \min$} & \multicolumn{2}{|c|}{$8 \min$} \\
\hline & Control & DCA & Control & DCA & Control & DCA \\
\hline ATP & $27.2 \pm 0.8$ & $26.9 \pm 1.1$ & $25.6 \pm 1.1$ & $26.9 \pm 1.5$ & $18.9 \pm 1.9$ & $22.6 \pm 1.6$ \\
\hline $\mathrm{PCr}$ & $78.0 \pm 3.0$ & $77.4 \pm 2.7$ & $26.9 \pm 4.9$ & $50.5 \pm 5.3^{*}$ & $7.1 \pm 1.8$ & $11.6 \pm 5.2$ \\
\hline $\mathrm{Cr}$ & $37.2 \pm 2.2$ & $38.1 \pm 3.1$ & $87.7 \pm 6.0$ & $65.1 \pm 6.2$ & $114.6 \pm 2.5$ & $105.2 \pm 7.0$ \\
\hline $\mathrm{AC}$ & $4.5 \pm 1.0$ & $11.8 \pm 1.3^{*}$ & $7.5 \pm 0.8$ & $10.8 \pm 1.2^{\ddagger}$ & $12.9 \pm 1.3$ & $15.8 \pm 2.0$ \\
\hline $\mathrm{La}$ & $18.6 \pm 0.7$ & $16.7 \pm 3.0$ & $55.9 \pm 7.3$ & $36.1 \pm 6.4^{\ddagger}$ & $73.6 \pm 8.5$ & $60.0 \pm 12.3$ \\
\hline Gly & $408 \pm 19$ & $434 \pm 19$ & $350 \pm 19$ & $379 \pm 27$ & $282 \pm 13$ & $335 \pm 30$ \\
\hline
\end{tabular}

Administration of DCA $(50 \mathrm{mg} / \mathrm{kg}$ body mass infused over $30 \mathrm{~min})$ or saline (control) before the onset of voluntary human skeletal muscle exercise with ischemia. $C r$, Free creatine; $A C$, acetylcarnitine; $L a$, lactate; and $G l y$, glycogen. Data are mean \pm SE. All units are $\mathrm{mmol} \cdot \mathrm{kg}^{-1}$ dry mass. ${ }^{\ddagger} P<0.05, * P<0.01$ indicate that the concentration recorded after DCA administration was significantly different from the control condition. 


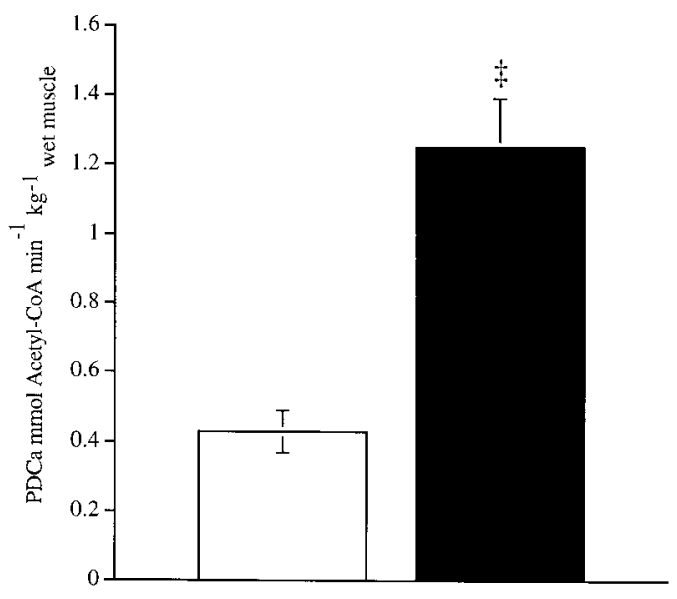

Figure 2. PDCa status in resting skeletal muscle after pretreatment with saline (open bar) or DCA (filled bar, $50 \mathrm{mg} \cdot \mathrm{kg}^{-1}$ body mass infused over $30 \mathrm{~min}) .{ }^{\ddagger} P<0.01$ indicates DCA significantly higher than the control visit. PDCa is expressed as $\mathrm{mmol}$ acetyl-CoA $/ \mathrm{min} / \mathrm{kg}$ wet muscle at $37^{\circ} \mathrm{C}$. Values are mean $\pm \mathrm{SE}$.

trol visit and $141 \pm 22.3 \mathrm{mmol}$ ATP $\cdot \mathrm{kg}^{-1} \mathrm{dm}$ in the DCA visit $(P=0.32)$. There was a net accumulation of acetylcarnitine in both visits, although this tended to be lower after DCA (Fig. 5).

\section{Discussion}

In this study we demonstrated that increasing the availability of acetyl groups in resting human skeletal muscle reduces, to a large extent, $\mathrm{PCr}$ degradation and lactate accumulation early during ischemic exercise but does not alter these metabolic parameters as the point of volitional fatigue is approached. Therefore, the accumulation of the metabolites associated with the development of skeletal muscle fatigue during ischemia (6, $18,25)$ can be delayed during voluntary exercise with DCA pretreatment. However, it remains to be determined whether

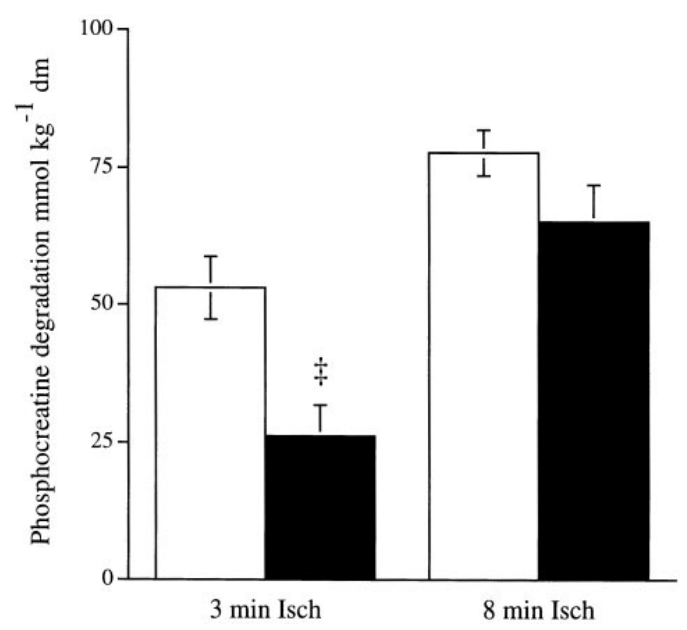

Figure 3. PCr degradation during human skeletal muscle contraction after pretreatment with saline (open bars) or DCA (filled bars, 50 $\mathrm{mg} \cdot \mathrm{kg}^{-1}$ body mass infused over $\left.30 \mathrm{~min}\right) .{ }^{\ddagger} P<0.01$ indicates DCA significantly lower than the control visit. Values are mean \pm SE.

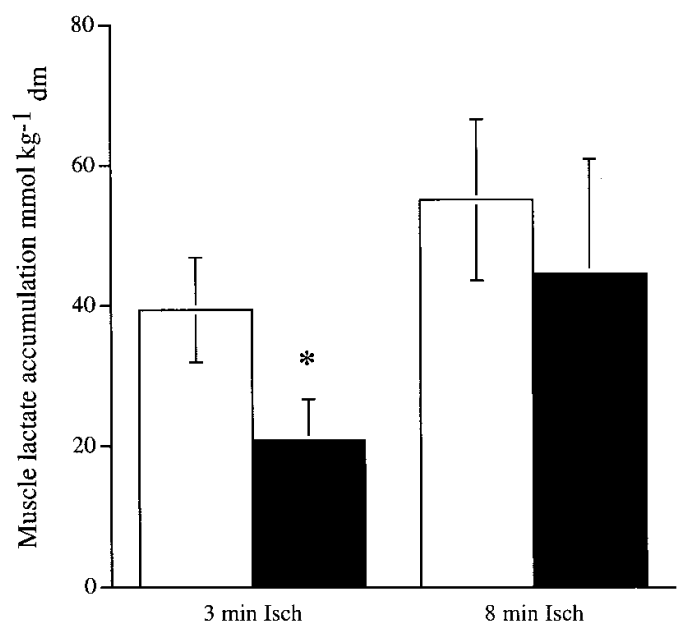

Figure 4. Muscle lactate accumulation during human skeletal muscle contraction after pretreatment with saline (open bars) or DCA (filled bars, $50 \mathrm{mg} \cdot \mathrm{kg}^{-1}$ body mass infused over $30 \mathrm{~min}$ ). $* P<0.05$ indicates DCA significantly lower than the control visit. Values are mean \pm SE.

this represents a strategy for improving the functional capacity of patients with compromised limb blood flow such as in peripheral vascular disease or heart failure (6-8).

$\mathrm{PCr}$ degradation and oxygen availability. At the onset of exercise, substrate level phosphorylation from $\mathrm{PCr}$ hydrolysis and glycolysis is the principal mechanism responsible for ATP regeneration. It has long been debated whether the extent of this so called "anaerobic" ATP regeneration reflects a lag in oxygen delivery to the contracting muscle or whether it is due to an intracellular biochemical limit to the rate of onset of oxygen utilization by the mitochondria $(26,27)$. Irrespective of the mechanism, this reliance on substrate level phosphorylation at the onset of exercise has been termed the oxygen deficit (26,

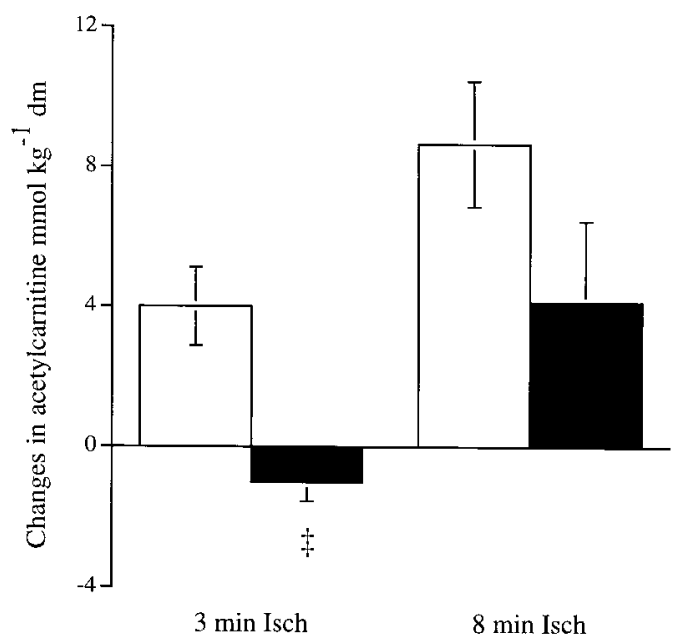

Figure 5. Changes in acetylcarnitine concentration during human skeletal muscle contraction after pretreatment with saline (open bars) or DCA (filled bars, $50 \mathrm{mg} \cdot \mathrm{kg}^{-1}$ body mass infused over $30 \mathrm{~min}$ ). ${ }^{\ddagger} P<$ 0.01 indicates DCA significantly different than the control group. Values are $\mathrm{mmol} \cdot \mathrm{kg}^{-1}$ dry (mass mean $\pm \mathrm{SE}$ ). 
$28,29)$, with the extent of the oxygen deficit being closely associated with the development of muscle fatigue (26).

At the onset of exercise, the kinetics of $\mathrm{PCr}$ degradation follow a similar pattern to $\mathrm{VO}_{2}$ kinetics (30). Further, at the onset of hypoxic exercise, the time constant for pulmonary $\dot{\mathrm{V}} \mathrm{O}_{2}$ kinetics is greater, thereby resulting in a greater oxygen deficit $(28,29)$ or a more marked $\mathrm{PCr}$ degradation $(31)$. These data have been interpreted to mean that muscle oxygen delivery is the major determinant of $\mathrm{PCr}$ degradation at the onset of exercise. Also, based on this interpretation, it would be expected that a more rapid change from rest to exercise would result in greater PCr degradation when assessed during steady state. When this hypothesis was tested, the rate of transition from rest to steady state did not alter the extent of skeletal muscle PCr degradation (32). In addition, although ischemia and hypoxia appear to result in greater PCr degradation (31, $33)$, there is also greater muscle recruitment under these conditions in order for steady state function to be maintained (33). Therefore, the lower PCr concentration during ischemia and hypoxia may simply reflect the extent of muscle activation rather than the effect of a reduction in muscle oxygen availability per se. It has also been demonstrated that when leg blood flow is modestly limited during exercise in normoxia, using lower-body positive pressure, pulmonary $\mathrm{V}_{2}$ kinetics are unaltered (34). This suggests that the effects of hypoxia on pulmonary $\dot{\mathrm{V}} \mathrm{O}_{2}$ kinetics $(28,29)$ do not reflect what occurs at the site of oxygen consumption, the exercising limb. Finally, Grassi et al. have demonstrated recently that the $\dot{\mathrm{V}} \mathrm{O}_{2}$ kinetics at the onset of exercise were not determined by blood flow to the contracting limb (27). However, they and others have been unable to determine whether intracellular metabolism or vascular perfusion heterogeneity was responsible for the rise in $\dot{\mathrm{V}} \mathrm{O}_{2}$ at the onset of exercise $(35,36)$. Overall, the above findings indicate that the kinetics of $\mathrm{PCr}$ degradation, at the onset of exercise, are not determined by bulk oxygen delivery.

We have established in a canine model of muscle ischemia that activation of PDC at rest and the subsequent accumulation of oxidative substrate, in the form of acetylcarnitine, substantially reduce muscle PCr degradation during a subsequent period of muscle contraction under conditions of identical oxygen delivery $(5,9)$. This provided the first direct evidence that a large proportion of the $\mathrm{PCr}$ degraded at the onset of skeletal muscle contraction is unrelated to oxygen availability but rather reflects an inherent lag in intracellular oxidative metabolism. In this study, we were able to demonstrate in human skeletal muscle that PDC activation at rest reduced the reliance on PCr stores by $\sim 50 \%$ during 3 min of ischemic exercise. Indeed, since there exists a proportionality between muscle oxygen consumption and PCr hydrolysis (10), then in this study a faster onset of oxidative phosphorylation must have occurred during the initial 3 min of exercise, after PDC activation. In support of this interpretation, muscle lactate accumulation was also reduced by $\sim 50 \%$.

During skeletal muscle contraction, the creatine kinase reaction is thought to be at or near equilibrium (37). Therefore, when the rate of ATP hydrolysis is increased by contraction the activators of glycogenolysis, ADP and AMP, increase transiently as ATP concentration is maintained relatively constant at the expense of the PCr stores $(10,37)$. However, as the concentration of $\mathrm{PCr}$ falls, muscle inorganic phosphate (Pi) accumulates to such an extent that it reduces the $K_{\mathrm{m}}$ of glycogen phosphorylase for AMP and hence increases glycolytic flux (for a comprehensive review of the regulation of glycogen metabolism see reference 38). Therefore, it becomes evident that if the rate of onset of oxidative phosphorylation is increased, the reduced reliance on $\mathrm{PCr}$ to rephosphorylate ADP will automatically result in a reduced rate of $\mathrm{Pi}$ accumulation, a reduced rate of glycolysis, and hence lower muscle lactate accumulation. In this study, glycogen utilization did not significantly differ between visits at any specific time point. However, it should be noted that there is normally a higher degree of variability in glycogen degradation between subjects when compared with other metabolites (20) and that there was a trend for an overall reduction in the rate of glycogen utilization $(F$ ratio $=13.3, P=0.02$, ANOVA $)$. This, coupled with the lower muscle lactate accumulation after $3 \mathrm{~min}$ of ischemic exercise, indicates that glycolytic flux during the initial period of exercise was indeed lower after DCA.

Models of muscle ischemia. This exercise model has been well characterized for investigating human skeletal muscle metabolism under ischemic conditions $(16,18,33,34)$. In this study, the metabolic effects of DCA were clearly evident after 3 min of ischemic exercise, with reductions in PCr degradation and lactate accumulation as well as a decline in acetylcarnitine concentration when compared with the control visit. After 8 min of exercise, however, DCA did not appear to alter the metabolic responses in the muscle. To discuss this observation we must first consider the models of skeletal muscle contraction that have been used $(5,9,25)$.

In the canine model, muscle fibers of the gracilis are stimulated to contract via electrical stimulation of the nerve to produce maximal twitch force, with all fiber types being recruited simultaneously (25). This results in $\sim 80 \%$ of the maximal oxidative flux under normal blood flow conditions. In the present study, the order of muscle fiber recruitment would have been that of the normal voluntary pattern (39). That is, the early contractile and metabolic profile of the voluntary knee extension exercise would have primarily reflected the contribution from type I muscle fibers, i.e., those from the smaller motor units. Critically, and unlike the situation observed with the canine twitch model, as the duration of ischemic exercise continues, a greater recruitment of muscle fibers would have occurred in order to maintain muscle force production, in the face of fatigue $(33,40)$. This has been observed previously using the same experimental model as the present study (33). The main consequence of the different muscle recruitment patterns is that after 8 min of voluntary exercise a near maximal effort was required and the work load would have exceeded the maximal oxidative capacity of the muscle (under the present, ischemic conditions). Hence, regardless of the rate of onset of oxidative metabolism, the muscle fibers would not have been able to continue to regenerate ATP oxidatively and as a consequence $\mathrm{PCr}$ concentration declined towards zero.

In addition, in the present experimental protocol, a 30-s rest interval was given to allow for a muscle biopsy to be taken at the 3-min time point. The physiological consequences of this are that the first 3 min would have functioned as a "warm-up" period which would increase $\dot{\mathrm{V}} \mathrm{O}_{2}$ kinetics, spare $\mathrm{PCr}$, and reduce $\mathrm{Pi}$ accumulation during a subsequent period of contraction $(35,36,41)$. In the context of the present study, the net effect would be that the magnitude of the differences between the control group and the DCA group would have been diminished.

Biochemical mechanisms for the present findings. It has 
been demonstrated in vitro that activation of the intramitochondrial dehydrogenases and PDC in particular can play an important role in the overall regulation of oxidative phosphorylation (42). In particular, it has been shown that when the concentration of NAD-linked substrates is low, dehydrogenase activity is a major limit to flux through oxidative phosphorylation. This limitation (or regulatory feature) is lost when substrates, such as pyruvate and 2-oxoglutarate, are in excess (42). Therefore, it could be argued that the high accumulation of acetylcarnitine in resting skeletal muscle, after DCA pretreatment $\left(\sim 10 \mathrm{mmol} \cdot \mathrm{kg}^{-1} \mathrm{dm}\right)$, provided a substrate store for the TCA cycle dehydrogenases and thereby was the mechanism responsible for the sparing of $\mathrm{PCr}$ during the initial 3 min of exercise. This conclusion is supported by the finding that there was a net decline in acetylcarnitine concentration during the first 3 min of exercise, after DCA pretreatment, and indicates that at least some of the "stockpile" of oxidative substrate was used early during exercise. This interpretation is supported by the observation that the rate of NADH accumulation (and hence flux through the intramitochondrial dehydrogenases) from rest to steady state is greater after DCA pretreatment (9). If the above interpretation is correct, then our previous canine studies $(5,9)$ and the present data from human subjects represent the first in vivo confirmation of the phenomenon observed previously in isolated mitochondrial preparations (42).

In conclusion, it appears that during the initial period of human skeletal muscle exercise the contribution made by oxidative phosphorylation to ATP regeneration is limited, in part, by the availability of acetyl-CoA and flux through the PDC reaction even during ischemic conditions. DCA's ability to enhance contractile function $(5,9)$ and to delay the extent of the metabolic disturbance during ischemic exercise, in this study, may have implications for the rate of development of skeletal muscle fatigue in patients with peripheral vascular disease and heart failure.

\section{Acknowledgments}

This study was supported by the DERA, Centre for Human Sciences, and by grants from the Swedish Medical Research Council (MFR 4494), Karolinska Institute research funds, and the Swedish National Centre for Research in Sports.

\section{References}

1. Ludvik, B., G. Peer, A. Berzlanovich, S. Stifter, and H. Graf. 1991. Effects of dichloroacetate and bicarbonate on haemodynamic parameters in healthy volunteers. Clin. Sci. 80:47-51.

2. Wells, P.G., G.W. Moore, D. Rabin, G.R. Wilkinson, J.A. Oates, and P.W. Stacpoole. 1980. Metabolic effects and pharmacokinetics of intravenously administered dichloroacetate in humans. Diabetologia. 19:109-113.

3. Carraro, F., S. Klein, J.I. Rosenblatt, and R.R. Wolfe. 1989. Effect of dichloroacetate on lactate concentration in exercising humans. J. Appl. Physiol. 66:591-597.

4. Ludvik, B., G. Mayer, S. Stifter, D. Putz, U. Barnas, and H. Graf. 1993. Effects of dichloroacetate on exercise performance in healthy volunteers. Eur. J. Physiol. 423:251-254.

5. Timmons, J.A., S.M. Poucher, D. Constantin-Teodosiu, V. Worrall, I.A. Macdonald, and P.L. Greenhaff. 1996. Increased acetyl group availability enhances contractile function of canine skeletal muscle during ischemia. J. Clin. Invest. 97:879-883.

6. Holm, J., P. Björntorp, and T. Scherstén. 1972. Metabolic activity in human skeletal muscle: effect of peripheral arterial insufficiency. Eur. J. Clin. Invest. 2:321-325.

7. Regensteiner, J.G., and W.R. Hiatt. 1995. Exercise rehabilitation for patients with peripheral arterial disease. Exercise Sport Sci. Rev. 23:1-24.
8. Wilson, J.R., J.L. Martin, D. Schwartz, and N. Ferraro. 1984. Exercise intolerance in patients with chronic heart failure: role of impaired skeletal muscle nutritive flow. Circulation. 69:1079-1085.

9. Timmons, J.A., S.M. Poucher, D. Constantin-Teodosiu, I.A. Macdonald, and P.L. Greenhaff. 1997. The metabolic responses from rest to steady state determine contractile function in ischemic skeletal muscle. Am. J. Physiol. 273: E233-E238.

10. Meyer, R.A. 1988. A linear model of muscle respiration explains monoexponential phosphocreatine changes. Am. J. Physiol. 254:C548-C553.

11. Stacpoole, P.W. 1989. The pharmacology of dichloroacetate. Metabolism. 38:1124-1144.

12. Lysiak, W., P.K. Lilly, P.P. Toth, and L.L. Bieber. 1988. Effect of the concentration of carnitine on acetylcarnitine production by rat heart mitochondria oxidizing pyruvate. Nutrition. 4:215-219.

13. Childress, C.C., B. Sacktor, and D.R. Traynor. 1966. Function of carnitine in the fatty acid oxidase-deficient insect flight muscle. J. Biol. Chem. 242: 754-760.

14. Harris, R.C., C.V.L. Foster, and E. Hultman. 1987. Acetylcarnitine formation during intense muscular contraction in humans. J. Appl. Physiol. 63: $440-442$.

15. Sahlin, K. 1989. Muscle carnitine metabolism during incremental dynamic exercise in humans. Acta Physiol. Scand. 138:259-262.

16. Eiken, O., and H. Bjurstedt. 1987. Dynamic leg exercise in man as influenced by experimental restriction of blood flow in the working muscles. Acta Physiol. Scand. 131:339-345.

17. Bjurstedt, H., and O. Eiken. 1995. Graded ischaemia in exercising human skeletal muscles: methods and applications. News Physiol. Sci. 10:193-197.

18. Sundberg, C.J., and L. Kaijser. 1992. Effects of graded restriction of perfusion on circulation and metabolism in the working leg: quantification of a human ischaemia model. Acta Physiol. Scand. 146:1-9.

19. Bergström, J. 1975. Percutaneous needle biopsy of skeletal muscle in physiological and clinical research. Scand. J. Clin. Lab. Invest. 35:609-616.

20. Harris, R.C., E. Hultman, and L.-O. Nordesjö. 1974. Glycogen, glycolytic intermediates and high energy phosphates determined in biopsy samples of musculus femoris of man at rest. Methods and variance values. Scand. J. Clin. Lab. Invest. 33:109-120.

21. Cederblad, G., J.I. Carlin, D. Constantin-Teodosiu, P. Harper, and E. Hultman. 1990. Radioiostopic assays of CoASH and carnitine and their acetylated forms in human skeletal muscle. Anal. Biochem. 185:274-278.

22. Constantin-Teodosiu, D., G. Cederblad, and E. Hultman. 1991. A sensitive radioisotopic assay of pyruvate dehydrogenase complex in human muscle tissue. Anal. Biochem. 198:347-351.

23. Snedecor, G.W., and W.G. Cochran. 1956. Statistical Methods. 5th ed. Iowa State University, Ames. 394-412.

24. Greenhaff, P.L., M.E. Nevill, K. Soderlund, K. Bodin, L.H. Boobis, C Williams, and E. Hultman. 1994. The metabolic responses of human type I and type II muscle fibres during maximal treadmill sprinting. J. Physiol. (Lond.). 478:149-155.

25. Timmons, J.A., S.M. Poucher, D. Constantin-Teodosiu, V. Worrall, I.A. Macdonald, and P.L. Greenhaff. 1996. The metabolic responses of canine gracilis muscle during contraction with partial ischemia. Am. J. Physiol. 270:E400E406.

26. Linnarsson, D., J. Karlsson, L. Fagraeus, and B. Saltin. 1974. Muscle metabolites and oxygen deficit with exercise in hypoxia and hyperoxia. J. Appl. Physiol. 36:399-402.

27. Grassi, B., D.C. Poole, R.S. Richardson, D.R. Knight, B.K. Erickson, and P.D. Wagner. 1996. Muscle $\mathrm{O}_{2}$ uptake kinetics in humans: implications for metabolic control. J. Appl. Physiol. 80:988-998.

28. Engelen, M., J. Porszasz, M. Riley, K. Wasserman, K. Maehara, and T.J. Barstow. 1996. Effects of hypoxic hypoxia on $\mathrm{O}^{2}$ uptake and heart rate kinetics during heavy exercise. J. Appl. Physiol. 81:2500-2508.

29. Murphy, P.C., L.A. Cuervo, and R.L. Hughson. 1989. A study of cardiorespiratory dynamics with step and ramp exercise tests in normoxia and hypoxia. Cardiovasc. Res. 23:825-832.

30. McCreary, C.R., P.D. Chilibeck, G.D. Marsh, D.H. Paterson, D.A. Cunningham, and R.T. Thompson. 1996. Kinetics of pulmonary oxygen uptake and muscle phosphates during moderate-intensity calf exercise. J. Appl. Physiol. 81: 1331-1338.

31. Katz, A., and K. Sahlin. 1987. The effects of decreased oxygen availability on NADH and lactate contents in human skeletal muscle during exercise. Acta Physiol. Scand. 131:119-127.

32. Sahlin, K., J.M. Ren, and S. Broberg. 1988. Oxygen deficit at the onset of submaximal exercise is not due to a delayed oxygen transport. Acta Physiol. Scand. 134:175-180.

33. Sundberg, C.J. 1994. Exercise and training during graded leg ischemia in healthy man. Acta Physiol. Scand. 150:2-50.

34. Williamson, J.W., P.B. Raven, and B.J. Whipp. 1996. Unaltered oxygen uptake kinetics at exercise onset with lower-body positive pressure in humans. Exp. Physiol. 81:695-705.

35. Laurent, D., B. Authier, J.F. Lebas, and A. Rossi. 1992. Effect of prior exercise in $\mathrm{Pi} / \mathrm{PC}$ ratio and intracellular $\mathrm{pH}$ during a standardized exercise. A study on human muscle using $\left[{ }^{31} \mathrm{P}\right]$ NMR. Acta Physiol. Scand. 144:31-38. 
36. Yoshida, T., J. Kamiya, and K. Hishimoto. 1995. Are oxygen uptake kinetics at the onset of exercise speeded up by local metabolic status in active muscles? Eur. J. Appl. Physiol. 70:482-486.

37. Roman, B.B, J.M. Foley, R.A. Meyer, and A.P. Koretsky. 1996. Contractile and metabolic effects of increased creatine kinase activity in mouse skeletal muscle. Am. J. Physiol. 270:C1236-C1245.

38. Connett, R.J., and K. Sahlin. 1996. Control of glycolysis and glycogen metabolism. In American Physiological Society Handbook of Physiology. Section 12: Exercise: Regulation and Integration of Multiple Systems. L.B. Rowell and J.T. Shepherd, editors. Oxford University Press, New York and Oxford. 870910 .
39. Henneman, E. 1957. Relationship between size of neurons and their susceptibility to discharge. Science. 126:1345-1347.

40. Moritani, T., W.M. Sherman, M. Shibata, T. Matsumoto, and M. Shinohara. 1992. Oxygen availability and motor unit activity in humans. Eur. J. Appl. Physiol. 64:552-556.

41. Bruton, J.D., H. Westerblad, A. Katz, and J. Lännergren. 1996. Aug mented force output in skeletal muscle fibers of Xenopus following a preceding bout of activity. J. Physiol. 493:211-217.

42. Moreno-Sanchez, R., B.A. Hogue, and R.G. Hansford. 1990. Influence of NAD-linked dehydrogenase activity on flux through oxidative phosphorylation. Biochem. J. 268:421-428. 